PROCEEDINGS OF THE

AMERICAN MATHEMATICAL SOCIETY

Volume 139, Number 6, June 2011, Pages 2067-2072

S 0002-9939(2010)10608-1

Article electronically published on November 5, 2010

\title{
A NOTE ON DENSITY FOR THE CORE OF UNBOUNDED BERGMAN OPERATORS
}

\author{
SHERWIN KOUCHEKIAN AND JAMES E. THOMSON
}

(Communicated by Michael T. Lacey)

\begin{abstract}
In this paper, we identify a large collection of open subsets of the complex plane for which the core of corresponding unbounded Bergman operators is densely defined. This result gives the necessary background to investigate the concept of invariant subspaces, index, and cyclicity in the unbounded case.
\end{abstract}

\section{INTRODUCTION}

Let $T$ denote a densely defined linear operator on a Hilbert space $\mathcal{H}$; that is, $T: \mathcal{D}(T) \rightarrow \mathcal{H}$, where the domain of $T$, given by $\mathcal{D}(T)=\{h \in \mathcal{H}: T h \in \mathcal{H}\}$, is dense in $\mathcal{H}$. For $n \geq 1$, define the iterates of $T$ by $T^{n}: \mathcal{D}\left(T^{n}\right) \rightarrow \mathcal{H}$, where

$$
\mathcal{D}\left(T^{n}\right)=\left\{h \in \mathcal{D}\left(T^{n-1}\right): T h \in \mathcal{D}(T)\right\}
$$

with the convention that $T^{0}=I d$ is the identity operator on $\mathcal{H}$.

Definition 1.1. Given a densely defined operator $T$ on $\mathcal{H}$, the core of $T$ is defined by

$$
\mathcal{D}_{T}=\bigcap_{n=1}^{\infty} \mathcal{D}\left(T^{n}\right)
$$

Whenever the operator $T$ is understood, we simply write $\mathcal{D}$ instead of $\mathcal{D}_{T}$.

It follows immediately that $T \mathcal{D} \subseteq \mathcal{D}$; i.e., $T$ leaves $\mathcal{D}$ invariant and, by definition, it must be the largest invariant set. Therefore, any definition for an invariant subspace of $T$ should be restricted to its core $\mathcal{D}_{T}$. It may very well happen that $\mathcal{D}_{T}$ is the trivial zero subspace for $T$ (necessarily unbounded); however, one would naturally like to have the other extreme. In other words, one wishes to work with densely defined operators such that their cores are also dense in the underlying Hilbert spaces. Of course, this question could only make sense when a particular operator is concerned.

To provide an easy, yet important, example, we consider the Fock space $F^{2}$. This is the space of all entire functions on the complex plane $\mathbb{C}$ such that their modulus are square integrable with respect to the gaussian measure $d \mu(z)=e^{-|z|^{2}} d A(z)$,

Received by the editors November 4, 2009 and, in revised form, May 31, 2010.

2010 Mathematics Subject Classification. Primary 32A36; Secondary 47B38, 32A35.

Key words and phrases. Unbounded Bergman operators, functional calculus, core of a Bergman operator.

(C)2010 American Mathematical Society Reverts to public domain 28 years from publication 
where $d A(z)=\frac{1}{\pi} d x d y$ is the normalized Lebesgue area measure. The operator of multiplication by the independent variable $z$ is defined by $\left(M_{z} f\right)(z)=z f(z)$ for all $f$ in $\mathcal{D}\left(M_{z}\right)=\left\{f \in F^{2}: z f \in F^{2}\right\}$. It follows that $M_{z}$ is an unbounded operator (see [2]). Moreover, using the power series expansion of an entire function $f(z)=\sum_{n=0}^{\infty} a_{n} z^{n}$ in $\mathbb{C}$, it is not hard to show (see [1) that the partial sums converge to $f$ in $F^{2}$. As a consequence, we have that the set of all polynomials is a dense linear manifold in the Fock space. Now from the definition of the core (1.2), one can easily see that polynomials are contained in $\mathcal{D}_{M_{z}}$. Therefore, $M_{z}$ has a dense core. The same question for the core of unbounded multiplication operators on the Bergman space is non-trivial. In fact, the main purpose of this paper is to prove the existence of a large collection of unbounded open subsets of the plane for which the core of the corresponding multiplication operator by the independent variable $z$ is dense. We also mention that this collection of open sets includes most natural cases such as simply connected regions, any open subset of a simply connected region, and much more. As a result, we will give the necessary condition for developing a fruitful invariant theory, where concepts such as the index of an invariant subspace and cyclicity can be extended from the bounded case to the unbounded situation (see Duren and Schuster [6] for definitions and obtained results in this regard for the theory of (bounded) Bergman operators).

Throughout this paper $G$ is assumed to be an open subset of the complex plane $\mathbb{C}$. Denote by $L^{2}(G)$ the Hilbert space of square integrable functions with respect to the Lebesgue area measure $d \mathcal{A}=d x d y$ restricted to $G$. The Bergman space $L_{a}^{2}(G)$ is the closed subspace of $L^{2}(G)$ whose elements are analytic functions on $G$. The algebra of all analytic functions on $G$ is denoted by $\operatorname{Hol}(G)$, and $H^{\infty}(G)$ is the space of bounded analytic functions on $G$. If $\varphi$ belongs to $\operatorname{Hol}(G)$ and $\mathcal{D}\left(M_{\varphi, G}\right)=$ $\left\{f \in L_{a}^{2}(G): \varphi f \in L^{2}(G)\right\}$, the operator of multiplication by $\varphi$ on $G$ is defined as $M_{\varphi, G}: f \mapsto \varphi f$ for $f$ in $\mathcal{D}\left(M_{\varphi, G}\right)$. In particular if $\varphi(z)=z, M_{z, G}$ is called the (unbounded) Bergman operator and it will be denoted by $S_{G}$. When there is no ambiguity regarding the open set $G, M_{\varphi, G}$ and $S_{G}$ are denoted by $M_{\varphi}$ and $S$, respectively.

The operator $M_{z}$ on the Fock space mentioned above and the multiplication operator $M_{\varphi, G}$, where $G$ is an unbounded open subset of the plane, belong to a larger class of operators which are called the unbounded subnormal operators. Significant progress in understanding these operators has been made during the recent past. The papers [4, 10, and [12 are a few to mention and contain further references on this topic. The study of unbounded Bergman operators has also been started in [4, 7], 8], and 9]. These papers deal particularly with some fundamental questions regarding the unbounded Bergman operators. The present work can be considered as a continuation of this study.

\section{Density PRoblem FOR THE CORE}

In what follows $\mathbb{D}$ is the open unit disc, $\partial \mathbb{D}$ stands for the unit circle, and $\mathbb{C}_{\infty}$ denotes the extended complex numbers. Our main goal is to prove the following result.

Theorem 2.1. Let $G$ be an open subset of the plane. If the component of the complement of $G$ with respect to $\mathbb{C}_{\infty}$ that contains the point at infinity does not 
equal the singleton $\{\infty\}$, then the Bergman operator $S$ defined on $G$ has a dense core in $L_{a}^{2}(G)$.

The proof of Theorem 2.1] uses the theory of Hardy spaces and univalent functions. Recall that an analytic function $f$ in $\mathbb{D}$ belongs to the Hardy space $H^{p}(\mathbb{D}), 0<$ $p<\infty$, if $\sup _{r<1}\left\{(2 \pi)^{-1} \int_{0}^{2 \pi}\left|f\left(r e^{i t}\right)\right|^{p} d t\right\}<\infty$. We will assume some basics from the theory of $H^{p}$ spaces.

Lemma 2.3 is the key factor in proving Theorem 2.1. This lemma and its corollary are also interesting in the theory of Hardy spaces and have not been observed before. In fact, since there is no advantage in restricting ourselves to the Hardy spaces, we prove Lemma 2.3 for a larger class of functions, the Nevanlinna class, which includes all the Hardy spaces $H^{p}(\mathbb{D})$. In brief, a non-zero analytic function $f$ in $\mathbb{D}$ belongs to the Nevanlinna class $N$ if and only if $f$ is the quotients of two bounded analytic functions, $f=g / h$, where $h$ has no zeros on $\mathbb{D}$. For other equivalent statements regarding functions in $N$, we refer to any classical work on this topic. Finally, recall also that an outer function on $\mathbb{D}$ is of the form

$$
F(z)=\alpha \exp \left(\frac{1}{2 \pi} \int_{0}^{2 \pi} \frac{e^{i t}+z}{e^{i t}-z} \log \omega\left(e^{i t}\right) d t\right)
$$

where $|\alpha|=1, \omega\left(e^{i t}\right)>0$ a.e. on the unit circle $\partial \mathbb{D}$, and $\log \omega\left(e^{i t}\right)$ is integrable with respect to the Lebesgue measure on $\partial \mathbb{D}$. We begin with a lemma.

Lemma 2.3. If $F$ is an outer function in $N$, then there is a bounded outer function $g$ on $\mathbb{D}$ such that $g F^{n} \in H^{\infty}(\mathbb{D})$ for all $n \geq 1$.

Proof. In order to prove the lemma, it is more convenient to regard $H^{p}(\mathbb{D}), 0<$ $p \leq \infty$, as a subspace of $L^{p}(\partial \mathbb{D})$. That is, we identify $H^{p}(\mathbb{D})$ with the set of boundary functions $f\left(e^{i \theta}\right)$ for $f$ in $H^{p}(\mathbb{D})$, and similar identification will be made regarding functions in the Nevanlinna class $N$ (see [5]). Throughout the proof $m$ is the normalized Lebesgue measure on $\partial \mathbb{D}$.

First assume that $|F(w)| \geq e$ a.e. $[m]$, and let $h(w)=\log |F(w)|$. Clearly $h(z) \geq 1$ a.e. $[m]$ and $h \in L^{1}(\partial \mathbb{D})$. Next, for $k=0,1,2, \ldots$, define

$$
A_{k}=\left\{w \in \partial \mathbb{D}: 2^{k} \leq h(w)<2^{k+1}\right\},
$$

and let

$$
\Phi(w)=\sum_{k=0}^{\infty} 2^{k} \mathcal{X}_{A_{k}}(w)
$$

where $\mathcal{X}_{A}(w)$ is the characteristic function for the set $A$. Using (2.4) and (2.5), it is easy to check that

$$
1 \leq \Phi(w) \leq h(w) \leq 2 \Phi(w) \quad \text { a.e }[m] .
$$

Consequently

$$
\sum_{k=0}^{\infty} 2^{k} m\left(A_{k}\right)=\int_{\partial \mathbb{D}} \Phi d m \leq \int_{\partial \mathbb{D}} h d m<\infty
$$

It is an elementary exercise that there exists a sequence of positive constants $\left\{c_{k}\right\}$ such that $c_{k} \rightarrow \infty$ and

$$
\sum_{k=0}^{\infty} 2^{k} c_{k} m\left(A_{k}\right)<\infty
$$


Now if we define $\Psi$ on $\partial \mathbb{D}$ by

$$
\Psi(w)=\sum_{k=0}^{\infty} 2^{k} c_{k} \mathcal{X}_{A_{k}}(w),
$$

inequality (2.7) implies that $\Psi \in L^{1}(\partial \mathbb{D})$. Thus from the standard construction of an outer function (see [5]), there exists a bounded outer function $g$ on $\mathbb{D}$ satisfying $|g(w)|=e^{-\Psi(w)}$ a.e. $[m]$. We will show that $g$ is the desired function. To see this, note that for any fixed $n \geq 1$

$$
\left|g(w) F^{n}(w)\right|=e^{n h(w)-\Psi(w)} .
$$

On the other hand, from (2.6) together with definitions of $\Phi$ and $\Psi$, we have

$$
\begin{aligned}
n h(w)-\Psi(w) & \leq 2 n \Phi(w)-\Psi(w) \\
& =\sum_{k=0}^{\infty} 2 n 2^{k} \mathcal{X}_{A_{k}}(w)-\sum_{k=0}^{\infty} 2^{k} c_{k} \mathcal{X}_{A_{k}}(w) \\
& =\sum_{k=0}^{\infty}\left(2 n-c_{k}\right) 2^{k} \mathcal{X}_{A_{k}}(w) \quad \text { a.e. }[m] .
\end{aligned}
$$

Since $\lim _{k \rightarrow \infty} c_{k}=\infty$, there is a positive constant $M$ such that $2 n-c_{k}<0$ whenever $k>M$. Therefore

$$
\sum_{k=M+1}^{\infty}\left(2 n-c_{k}\right) 2^{k} \mathcal{X}_{A_{k}}(w) \leq 0 \quad \text { a.e. }[m],
$$

and as a result

$$
\begin{aligned}
n h(w)-\Psi(w) & \leq \sum_{k=0}^{M}\left(2 n-c_{k}\right) 2^{k} \mathcal{X}_{A_{k}}(w) \\
& \leq \sum_{k=0}^{M}\left(2 n-c_{k}\right) 2^{k} \quad \text { a.e. }[m] .
\end{aligned}
$$

Putting all together, we have shown that for a fixed $n \geq 1$

$$
\left|g(w) F^{n}(w)\right| \leq \exp \left(\sum_{k=0}^{M}\left(2 n-c_{k}\right) 2^{k}\right) \quad \text { a.e. }[m],
$$

where $M$ only depends on $n$. Since $n$ is arbitrary, we conclude that if $|F(w)| \geq e$ a.e. on $\partial \mathbb{D}, g F^{n} \in H^{\infty}(\mathbb{D})$ for all $n \geq 1$.

Next assume that $F$ is an arbitrary outer function in $N$. Let

$$
\Upsilon(w)=\left\{\begin{array}{cll}
e^{-1}|F(w)| & \text { if } & |f(w)|<e \\
1 & \text { if } & |f(w)| \geq e .
\end{array}\right.
$$

It follows that $\Upsilon(w) \leq 1$ a.e. $[m]$. Moreover since $\log |F| \in L^{1}(\partial \mathbb{D}), \log |\Upsilon| \in$ $L^{1}(\partial \mathbb{D})$. Thus, from the standard $H^{p}$ theory, one can find a bounded outer function $h$ such that $|h(w)|=\Upsilon(w)$ a.e. $[m]$. Now since the quotient of outer functions is again an outer function, it follows that $F / h$ is an outer function in $N$ satisfying $|F(w) / h(w)| \geq e$ a.e. $[m]$. Therefore, by a similar argument as in the first part of the proof, there exists an outer function $g$ in $H^{\infty}(\mathbb{D})$ such that

$$
\frac{g F^{n}}{h^{n}} \in H^{\infty}(\mathbb{D}) \text { for all } n \geq 1 \text {. }
$$


Finally noting that $h^{n} \in H^{\infty}(\mathbb{D})$, we conclude that $g F^{n} \in H^{\infty}(\mathbb{D})$ for all $n \geq 1$. This completes the proof of the lemma.

Observe that if $F$ is an outer function in $N, 1 / F$ is also outer and belongs to $N$. Thus we have also obtained the following result.

Corollary 2.8. If $F$ is an outer function in $N$, then there is an outer function $g$ in $H^{\infty}(\mathbb{D})$ such that $g F^{n} \in H^{\infty}(\mathbb{D})$ for all $n \in \mathbb{Z}$.

Our next proposition is in fact about the density of the core of the multiplication operator by an outer function. Moreover, it provides the last needed tool in proving our main result.

Proposition 2.9. If $\Omega$ is an open subset of $\mathbb{D}$ and if $F$ is an outer function in $N$, then the $\mathcal{D}_{F}=\bigcap_{n=0}^{\infty} \mathcal{D}\left(M_{F^{n}}\right)$ is dense in $L_{a}^{2}(\Omega)$.

Proof. Let $g$ be the bounded outer function in accordance with Lemma 2.3. In Rubel and Shields [11, see Theorem 5.1] it is shown that if $F$ is a bounded outer function, then the set $\left\{F h: h \in H^{\infty}(\mathbb{D})\right\}$ is weak ${ }^{*}$ sequentially dense in $H^{\infty}(\mathbb{D})$. Thus, in our case, there exists a sequence $\left\{h_{n}\right\}$ in $H^{\infty}(\mathbb{D})$ such that $g h_{n} \rightarrow 1$ weak $^{*}$ in $L^{\infty}(\mathbb{D})$, or equivalently

$$
\sup _{n \geq 1}\left\|g h_{n}\right\|_{\infty}<\infty \text { and } g(z) h_{n}(z) \rightarrow 1 \text { for all } z \text { in } \mathbb{D} \text {. }
$$

Now let $f$ be a non-zero function in $L_{a}^{2}(\Omega)$, and define $g_{n}$ on $\Omega$ by $g_{n}=g h_{n} f$. It follows from Lemma 2.3 that $g_{n} \in \bigcap_{k=0}^{\infty} \mathcal{D}\left(M_{F^{k}}\right)$ for all $n \geq 1$. Furthermore

$$
\left\|g_{n}-f\right\|_{L_{a}^{2}(\Omega)}^{2}=\int_{\Omega}\left|g(z) h_{n}(z)-1\right|^{2}|f(z)|^{2} d \mathcal{A}(z)
$$

The Dominated Convergence Theorem together with (2.10) implies that $g_{n} \rightarrow f$ in $L_{a}^{2}(\Omega)$. This proves the proposition.

Remark 2.11. From Definition 1.1. one can easily check that $M_{\varphi}^{n}=M_{\varphi^{n}}$; that is, for $\varphi \in \operatorname{Hol}(G), \mathcal{D}\left(M_{\varphi}^{n}\right)=\mathcal{D}\left(M_{\varphi^{n}}\right)$ and $M_{\varphi}^{n}$ agrees with $M_{\varphi^{n}}$, the operator of multiplication by $\varphi^{n}$ (see also [7]).

Proof of Theorem 2.1. Denote by $E$ the component of the complement of $G$ with respect to $\mathbb{C}_{\infty}$ that contains the point at infinity, and let $\Lambda=\mathbb{C}_{\infty} \backslash E$. It follows that $\Lambda$ is a region in $\mathbb{C}_{\infty}, G \subseteq \Lambda$ and, since by assumption $E$ consists of more than one point, $\Lambda$ is a simply connected region in $\mathbb{C}$ which is not the whole plane. By the Riemann Mapping Theorem, for a fixed point $a$ in $G$, there is a unique conformal mapping from $\mathbb{D}$ onto $\Lambda, \varphi: \mathbb{D} \rightarrow \Lambda$, such that $\varphi(0)=a$ and $\varphi^{\prime}(0)>0$. Now if we put $\Omega=\varphi^{-1}(G)$, it follows that $\Omega$ is an open subset of the unit disc.

Next define $U: L_{a}^{2}(G) \rightarrow L_{a}^{2}(\Omega)$ by $(U f)(z)=\varphi^{\prime}(z) f(\varphi(z))$ for all $f \in L_{a}^{2}(G)$. Since $\varphi$ is one-to-one and analytic on $\Omega$, it is well known that the mapping $U$ is an isometric isomorphism of $L_{a}^{2}(G)$ onto $L_{a}^{2}(\Omega)$ (see [3]). Furthermore, from the equality

$$
\left\|w^{n} f\right\|_{L_{a}^{2}(G)}^{2}=\int_{G}\left|w^{n} f\right|^{2} d u d v=\int_{\Omega}\left|\varphi^{n} f(\varphi(z))\right|^{2}\left|\varphi^{\prime}\right|^{2} d x d y=\left\|\varphi^{n} U f\right\|_{L_{a}^{2}(\Omega)}^{2}
$$

along with Remark 2.11, one easily sees that

$$
U\left[\mathcal{D}\left(S_{G}^{n}\right)\right]=\mathcal{D}\left(M_{\varphi^{n}, \Omega}\right) \quad \text { for all } n \geq 1 .
$$


Now in view of (2.12) and the fact that $U$ is an isomorphism, we conclude that the core of $S, \mathcal{D}_{S}=\bigcap_{n=0}^{\infty} \mathcal{D}\left(S^{n}\right)$, is dense in $L_{a}^{2}(G)$ if and only if the the core of $M_{\varphi}$, $\mathcal{D}_{M_{\varphi}}=\bigcap_{n=0}^{\infty} \mathcal{D}\left(M_{\varphi}^{n}\right)$, is dense in $L_{a}^{2}(\Omega)$. So the proof is complete by showing that the latter equivalent statement holds. Letting $\psi(z)=\varphi(z)-a$, if necessary, and observing that $\mathcal{D}\left(M_{\psi}^{n}\right)=\mathcal{D}\left(M_{\varphi}^{n}\right)$, we may assume that $\varphi(0)=0$.

Recall that if $p>0$ and $f \in H^{p}(\mathbb{D})$, then $f$ has a unique factorization of the form $f(z)=B(z) S(z) F(z)$, where $B(z)$ is a Blaschke product, $S(z)$ is a singular inner function, and $F(z)$ is an outer function in $H^{p}(\mathbb{D})$ defined as in (2.2) (see [5], Theorem 2.8). It is also known that if $f$ is one-to-one and analytic in $\mathbb{D}$, then $f \in H^{p}$ for all $p<\frac{1}{2}$ and the singular inner factor of $f$ is constantly 1 ([5], Theorems 3.16 and 3.17). Hence, since $\varphi(0)=0, \varphi$ is one-one and analytic

$$
\varphi(z)=z F(z)
$$

where $F$ is an outer function in $H^{p}$ for all $p<\frac{1}{2}$. But (2.13) implies that $\mathcal{D}\left(M_{F}^{n}\right) \subseteq$ $\mathcal{D}\left(M_{\varphi}^{n}\right)$, for all $n \geq 1$, and consequently $\mathcal{D}_{F} \subseteq \mathcal{D}_{\varphi}$. Now the theorem follows from Proposition 2.9.

\section{REFERENCES}

[1] V. Bargmann, "On a Hilbert space of analytic functions and an associated integral transform", Comm. Pure Appl. Math. 14 (1961), 187-214. MR0157250 (28:486)

[2] C. Beneteau, B. J. Carswell, and S. Kouchekian, "Extremal problems in the Fock space", Computational Methods and Function Theory 10 (2010), 189-206. MR2676450

[3] S. Bergman, The Kernel Function and Conformal Mapping, Amer. Math. Soc. Surveys and Monographs 5, Providence, RI (1950). MR0038439 (12:402a)

[4] J. B. Conway, K. H. Jin, and S. Kouchekian, "On Unbounded Bergman Operators", J. Math. Anal. Appl. 279 (2003), 418-429. MR1974034(2004b:47030)

[5] P. Duren, Theory of $H^{p}$ Spaces, Academic Press, New York (1970). MR0268655 (42:3552)

[6] P. Duren and A. Schuster, Bergman Spaces, American Mathematical Society, Providence, RI (2004). MR.2033762(2005c:30053)

[7] S. Kouchekian, "The Density Problem for Unbounded Bergman Operators", Integral Equations and Operator Theory 45 (2003), 319-342. MR1965338 (2004d:47065)

[8] S. Kouchekian and J. E. Thomson, "The Density Problem for Self-Commutators of Unbounded Bergman Operators", Integral Equations Operator Theory 52 (2005), 135-147. MR2138701 (2006a:47029)

[9] S. Kouchekian and J. E. Thomson, "On self-commutator of Toeplitz operators with rational symbols", Studia Math. 179, no. 1 (2005), 41-47. MR2291722 (2007m:47060)

[10] G. McDonald, and C. Sundberg, "On the Spectra of Unbounded Subnormal Operators", Canad. J. Math. 38 (1986), 1135-1148. MR869718 (87m:47058)

[11] L. A. Rubel and A. L. Shields, "The Space of Bounded Analytic Functions on a Region", Ann. Inst. Fourier (Grenoble) 16 (1966), 235-277. MR0198281(33:6440)

[12] J. Stochel and F. H. Szafraniec, "On Normal Extensions of Unbounded Operators. I", J. Operator Theory 14 (1985), 31-55. MR789376 (87d:47034)

Department of Mathematics \& Statistics, University of South Florida, Tampa, FLORIDA 33620-5700

E-mail address: skouchekian@usf.edu

Department of Mathematics, Virginia Tech, Blacksburg, Virginia 24061-0123

E-mail address: jthomson@math.vt.edu 